CTPU-17-16

OU-HEP-170421

\title{
Prospects for axion detection in natural SUSY with mixed axion-higgsino dark matter: back to invisible?
}

\author{
Kyu Jung Bae调, Howard Baer讯, and Hasan Serce䗆 \\ ${ }^{1}$ Center for Theoretical Physics of the Universe, \\ Institute for Basic Science (IBS), Daejeon 34051, Korea \\ ${ }^{2}$ Dept. of Physics and Astronomy, \\ University of Oklahoma, Norman, OK 73019, USA
}

\begin{abstract}
Under the expectation that nature is natural, we extend the Standard Model to include SUSY to stabilize the electroweak sector and PQ symmetry to stabilize the QCD sector. Then natural SUSY arises from a Kim-Nilles solution to the SUSY $\mu$ problem which allows for a little hierarchy where $\mu \sim f_{a}^{2} / M_{P} \sim 100-300 \mathrm{GeV}$ while the SUSY particle mass scale $m_{\mathrm{SUSY}} \sim 1-10 \mathrm{TeV} \gg \mu$. Dark matter then consists of two particles: a higgsino-like WIMP and a SUSY DFSZ axion. The range of allowed axion mass values $m_{a}$ depends on the mixed axion-higgsino relic density. The range of $m_{a}$ is actually restricted in this case by limits on WIMPs from direct and indirect detection experiments. We plot the expected axion detection rate at microwave cavity experiments. The axion-photon-photon coupling is severely diminished by charged higgsino contributions to the anomalous coupling. In this case, the axion may retreat, at least temporarily, back into the regime of near invisibility. From our results, we urge new ideas for techniques which probe both deeper and more broadly into axion coupling versus axion mass parameter space.
\end{abstract}

*Email: kyujungbae@ibs.re.kr

${ }^{\dagger}$ Email: baer@nhn.ou.edu

†Email: serce@ou.edu 


\section{Introduction}

It seems to be a tautology that nature is natural [1]. In particle physics, a working definition of naturalness is that each independent contribution to an observable ought to be comparable to or less than its measured value. For if one contribution were far greater, then some other supposedly unrelated contribution would need to be fine-tuned to exactly the right opposite-sign value such as to maintain the measured value. Such a situation is considered highly implausible or unnatural. In many circumstances, naturalness has turned out to be a reliable guide towards the correct laws of nature [2] while the presence of fine-tuning acts as a sieve to filter out faulty theories.

An example from the QCD sector of the Standard Model (SM) arises from 't Hooft's [3] solution to the old $U(1)_{A}$ problem [4] via the discovery of the $\theta$ vacuum. A consequence of the instanton-induced $\theta$ vacuum is that the QCD Lagrangian should contain an additional term

$$
\mathcal{L}_{\mathrm{QCD}} \ni \frac{\alpha_{s} \bar{\theta}}{8 \pi} G_{\mu \nu A} \tilde{G}_{A}^{\mu \nu}
$$

(where $G_{\mu \nu A}$ is the gluon field strength tensor) which gives rise to strong $C P$ violating interactions. The term $\bar{\theta}$ contains two separate contributions $\bar{\theta}=\theta_{\mathrm{QCD}}+\operatorname{Arg}\left[\operatorname{det}\left(m_{q}\right)\right]$. Measurements of the neutron EDM require $\bar{\theta} \lesssim 10^{-10}$. The strong CP problem of QCD- why $\bar{\theta}$ is so small- is thus a problem of naturalness. So far, the most compelling solution to the strong CP problem is to impose an additional (spontaneously broken) global Peccei-Quinn (PQ) symmetry which requires the existence of an (invisible) axion [5, 6, 7, 8, 9].

In the electroweak (EW) sector of the SM, it is well known that the Higgs boson mass is quadratically unstable under quantum corrections. Increasingly implausible fine-tunings are necessary to maintain the Higgs mass at its measured value $m_{h} \simeq 125 \mathrm{GeV}$ [10, 11] depending on the cut-off scale $\Lambda$ which demarcates the upper energy range of validity of the theory. A simple and elegant solution to the Higgs naturalness problem is to extend the underlying Poincaré spacetime symmetries to include their maximal structure: supersymmetry (SUSY). The minimal supersymmetrized Standard Model, or MSSM [12], is free of quadratic divergences but phenomenologically requires inclusion of soft SUSY breaking terms not too far from the weak scale. The MSSM predicts the existence of a panoply of new superpartner matter states: squarks, sleptons, gluinos, charginos and neutralinos. So far, superpartners have yet to be found at LHC [13, 14]; this has led to concern that the fine-tuning may creep back into the MSSM via log instead of quadratic divergences [1]. A Little Hierarchy problem (LHP) has emerged [15]: how can the weak scale as characterized by $m_{W, Z, h} \sim 100 \mathrm{GeV}$ be stable while superpartners apparently lie in the multi-TeV range?

Some perspective on the LHP can be gained by examining the scalar potential minimization conditions which relate the measured value of $m_{Z}$ to MSSM Lagrangian parameters:

$$
\begin{aligned}
\frac{m_{Z}^{2}}{2} & =\frac{m_{H_{d}}^{2}+\Sigma_{d}^{d}-\left(m_{H_{u}}^{2}+\Sigma_{u}^{u}\right) \tan ^{2} \beta}{\tan ^{2} \beta-1}-\mu^{2} \\
& \simeq-m_{H_{u}}^{2}-\Sigma_{u}^{u}-\mu^{2},
\end{aligned}
$$

where the latter approximate equality arises for $\tan \beta \gtrsim 3$. The $m_{H_{u, d}}^{2}$ are weak scale soft SUSY breaking Higgs mass terms, $\mu$ is the superpotential Higgs/higgsino mass parameter and 
$\tan \beta \equiv v_{u} / v_{d}$ is the ratio of Higgs field vevs. The terms $\Sigma_{u}^{u}$ and $\Sigma_{d}^{d}$ contain an assortment of 1-loop corrections; expressions can be found in the Appendix of Ref. [16]. For weak scale naturalness with less than $3 \%$ fine-tuning, from Eq. 2 we see that

1. $\mu \sim 100-350 \mathrm{GeV}$ [17] (the lower bound arises due to LEP2 searches for charginos),

2. $m_{H_{u}}^{2} \sim-(100-350)^{2} \mathrm{GeV}^{2}$ and

3. $\Sigma_{u}^{u}(i) \sim(100-350)^{2} \mathrm{GeV}^{2}$

(where $i$ labels the various loop contributions to $\Sigma$ ). In the case of $m_{H_{u}}^{2}$ : even if soft terms are at multi-TeV level at energy scale $Q \sim m_{\mathrm{GUT}}, m_{H_{u}}^{2}$ can be driven to natural values [18] at the weak scale thanks to the large top Yukawa coupling. This situation is known as radiatively driven naturalness. The $\Sigma_{u}^{u}$ term is typically dominated by the top squark contributions. It is minimized for TeV-scale highly mixed top-squarks- which are just the right conditions to lift $m_{h}$ up to $\sim 125 \mathrm{GeV}$ [19]. Mass limits on sparticles from requiring

$$
\Delta_{\mathrm{EW}} \equiv \mid \max \text { term on RHS of Eq. } 2 /\left(m_{Z}^{2} / 2\right)<30
$$

allow for $m_{\tilde{g}} \lesssim 5-6 \mathrm{TeV}$ so SUSY maintains naturalness even in the face of LHC measurements of $m_{h}$ and limits on sparticle masses [16, 20].

An important consequence of $\mu \sim 100-350 \mathrm{GeV}$ is that light higgsinos $\widetilde{W}_{1}^{ \pm}$and $\widetilde{Z}_{1,2}$ should exist. The lightest SUSY particle $\widetilde{Z}_{1}$ is a higgsino-like neutralino which is thermally underproduced as dark matter [21]. In our setup, where we also require an axion to solve the strong CP problem, then axions also make up a (potentially dominant) portion of the dark matter. The axion field is now just one element of an axion supermultiplet

$$
A=(s+i a) / \sqrt{2}+\sqrt{2} \theta \tilde{a}+\theta^{2} \mathcal{F}_{a}
$$

where $\theta$ now are superspace co-ordinates, $s$ is the $R$-parity-even spin-0 saxion field and $\tilde{a}$ is the $R$-parity-odd spin-1/2 axino field and $\mathcal{F}_{a}$ is the auxiliary field of the axion supermultiplet. In gravity-mediated SUSY breaking, one expects $m_{s}$ of order the gravitino mass $m_{3 / 2} \sim \mathrm{TeV}$ (similarly to other scalar masses). The axino mass $m_{\tilde{a}}$ is also typically of the same order $\left(\sim m_{3 / 2}\right)$ although it can be different in some models [22, 23, 24, 25]. In this case, the higgsinolike WIMPs can be non-thermally produced [26] due to production and late decays of axinos and saxions in the early universe [27, 28].

An important element of the LHP is to try to understand why $\mu \sim m_{W, Z, h} \ll m_{\text {SUSY }} \sim 1-10$ TeV. The SUSY version of the DFSZ [8] axion model offers an elegant solution known as the Kim-Nilles (KN) mechanism [29]. In KN, the Higgs and matter superfields actually carry PQ charge assignments with $Q_{\mathrm{PQ}}\left(H_{u}\right)+Q_{\mathrm{PQ}}\left(H_{d}\right)=-(n+1)$ (and various possibilities occur for PQ charge assignments for matter superfields). In this case, the $\mu$ term in the superpotential is forbidden which explains why $\mu$ is not of order the reduced Planck mass $M_{P} \sim 2.4 \times 10^{18}$ $\mathrm{GeV}$. However, now one may introduce a SM singlet but PQ charged chiral superfield $S$ with $Q_{\mathrm{PQ}}(S)=+1$ with non-renormalizable superpotential

$$
W \ni \lambda_{\mu} S^{n+1} H_{u} H_{d} / M_{P}^{n}
$$


Once the PQ symmetry is spontaneously broken, then the PQ fields develop a vev $\langle S\rangle \sim f_{a}$ where $f_{a}$ is the axion decay constant and sets the PQ scale. A $\mu$ term develops with

$$
\mu \sim \lambda_{\mu} f_{a}^{n+1} / M_{P}^{n}
$$

For $\mu \sim 100 \mathrm{GeV}, n=1$ and $\lambda_{\mu} \sim 1$, then one expects $f_{a} \sim 10^{10} \mathrm{GeV}$. For higher $n$ values, then much larger values of $f_{a}$ are expected. The value of $\mu$ here may be compared with the expected value of gravitino mass from simple supergravity models: $m_{3 / 2} \sim m_{\text {hidden }}^{2} / M_{P}$ (where $m_{\text {hidden }}$ denotes the mass scale associated with the hidden sector). Then the Little Hierarchy $\mu \ll m_{3 / 2}$

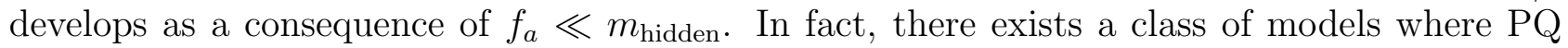
symmetry is broken radiatively as a consequence of SUSY breaking. In these radiatively-broken PQ models, it is typical to develop $\mu \sim 100 \mathrm{GeV}$ as a consequence of $m_{\mathrm{SUSY}} \sim 1-10 \mathrm{TeV}[30$.

In previous papers [31, 32, we have examined prospects for direct and indirect detection of the higgsino-like WIMPs from mixed axion-higgsino dark matter as expected in natural SUSY models. In the present paper, we examine prospects for axion detection at microwave cavity detectors such as ADMX [33, 34]. As with WIMP detection, our answer depends now not only on the axion mass and coupling, but also on the relative portion of dark matter made up of axions vis-a-vis WIMPs. As such, in Sec. 2, we examine the WIMP vs. axion relic density in an updated natural SUSY benchmark model. An issue which arises anew here is that, for a particular set of PQ parameters, if the WIMP abundance is enhanced compared to axions, then the particular parameter set may become ruled out by WIMP direct or indirect detection search limits. In Sec. 3, we examine prospects for axion detection in the SUSY DFSZ model at microwave cavity experiments. We re-evaluate the axion coupling $g_{a \gamma \gamma}$ and find that it is severely diminished from the non-SUSY DFSZ model or the KSVZ model due to the circulation of $\mathrm{PQ}$-charged higgsinos in the loop. Even so, a rather large range of $m_{a}$ values emerge as possible search targets. The range is somewhat disjoint due to the interplay between non-thermal WIMP production via axino or saxion production and decay. In Sec. 4. we summarize and present conclusions. In the SUSY DFSZ model, which we feel is most compelling due to simultaneously solving 1 . the gauge hierarchy problem, 2 . the strong CP problem, 3. the Little Hierarchy problem and 4. the SUSY $\mu$ problem, the discovery of the axion may require much deeper probes of axion coupling and much broader scans over axion mass. If not, the axion may retreat back into invisibility, at least in the near term future.

\section{Relic density of axions and higgsino-like WIMPs}

In this Section, we compute the relic abundance of higgsino-like WIMPs and DFSZ axions from an updated natural SUSY benchmark model. An older SUSY benchmark model dubbed SUA in Ref. 35] with $m_{\tilde{g}}=1.8 \mathrm{TeV}$ has apparently been excluded by recent LHC searches which now require $m_{\tilde{g}} \gtrsim 2 \mathrm{TeV}[13$, 14]. The new natural SUSY benchmark point from the two-extra-parameter non-universal Higgs model [36] (NUHM2) has parameters $m_{0}=5.3 \mathrm{TeV}$, $m_{1 / 2}=2.03 \mathrm{TeV}, A_{0}=-9.85 \mathrm{TeV}, \tan \beta=9$ with $\left(\mu, m_{A}\right)=(150,3000) \mathrm{GeV}$. This gives rise to a gluino mass $m_{\tilde{g}}=4.48 \mathrm{TeV}$, well above the reach of HL-LHC but within reach of a $33 \mathrm{TeV}$ LHC energy upgrade [37]. The value of $\Delta_{\mathrm{EW}}=29$ for $3.3 \% \mathrm{EW}$ fine-tuning. The thermally produced higgsino relic abundance is $\Omega_{\widetilde{Z}_{1}}^{\mathrm{TP}} h^{2}=0.006$ or about $5 \%$ of the measured dark matter abundance. The higgsino-like WIMP has mass $m_{\widetilde{Z}_{1}}=150.4 \mathrm{GeV}$. 


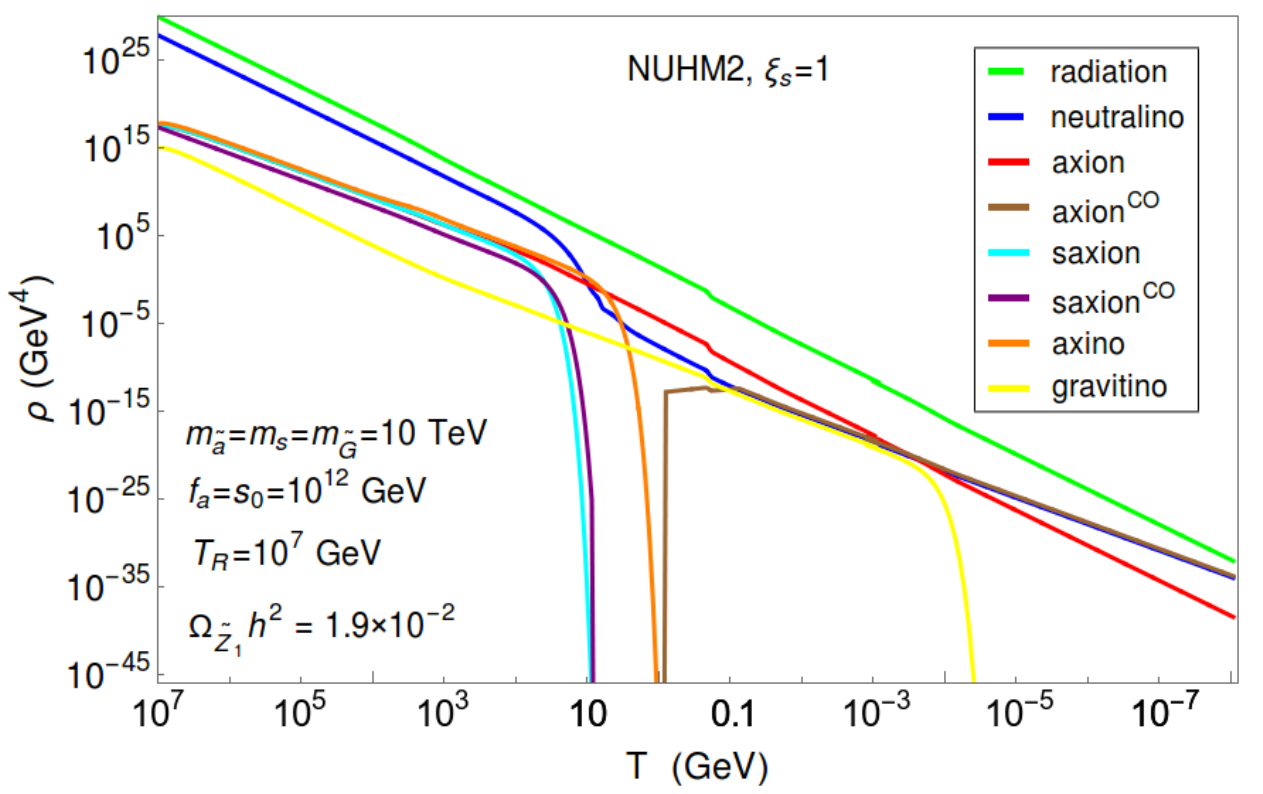

Figure 1: A plot of various energy densities $\rho$ vs. temperature $T$ starting from $T_{R}=10^{7} \mathrm{GeV}$ until the era of entropy conservation from our eight-coupled Boltzmann equation solution to the mixed axion-neutralino relic density in the SUSY DFSZ model for a natural SUSY benchmark point. We take $\xi_{s}=1$.

To evaluate the mixed neutralino-axion relic density, we apply the eight-coupled-Boltzmann equation computer code developed in Ref's [35, 38]. Starting from the time of re-heat with temperature $T_{R}$ at the end of the inflationary epoch, the computer code tracks the coupled abundances of radiation (i.e. SM particles), neutralinos, axinos, gravitinos, saxions and axions (the latter two consist of thermal/decay-produced components and coherent oscillations (CO)).

The CO abundance of axions is determined by its initial misalignment angle $\theta_{i}$ [39, 40]. For numerical analyses, we adopt a simple formula

$$
\Omega_{a}^{\mathrm{CO}} h^{2} \simeq 0.23 f\left(\theta_{i}\right) \theta_{i}^{2}\left(\frac{f_{a} / N_{\mathrm{DW}}}{10^{12} \mathrm{GeV}}\right)^{7 / 6}
$$

where $f\left(\theta_{i}\right)=\left[\log \left(\left(e /\left(1-\theta_{i}^{2} / \pi^{2}\right)\right)\right]^{7 / 6}\right.$ is the anharmonicity factor [40]. Provided the neutralino and thermal/decay-produced axion abundance is below the total measured DM abundance, the value of $\theta_{i}$ can always be adjusted so that CO-produced axions make up the remainder.

A plot of the energy densities vs. $T$ is shown for the SUSY DFSZ axion model for our natural SUSY NUHM2 benchmark model is shown in Fig 1. We take $T_{R}=10^{7} \mathrm{GeV} 1$ and $f_{a}=s_{0}=10^{12} \mathrm{GeV}$. We also take $m_{\tilde{a}}=m_{s}=m_{3 / 2}=10 \mathrm{TeV}$. The blue curve denotes the neutralino abundance which freezes out at $T \sim 10 \mathrm{GeV}$. Saxions decay around $T \sim 10 \mathrm{GeV}$ whilst axinos decay around $T \sim 1 \mathrm{GeV}$, the temperature also where axions start to oscillate.

\footnotetext{
${ }^{1}$ This value of $T_{R}$ is in accord with baryogenesis mechanisms such as non-thermal or Affleck-Dine leptogenesis [1].
} 


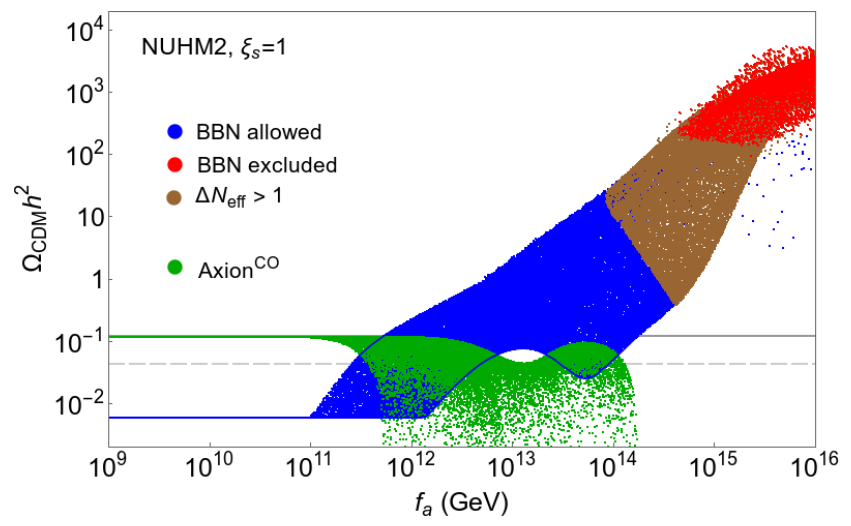

(a)

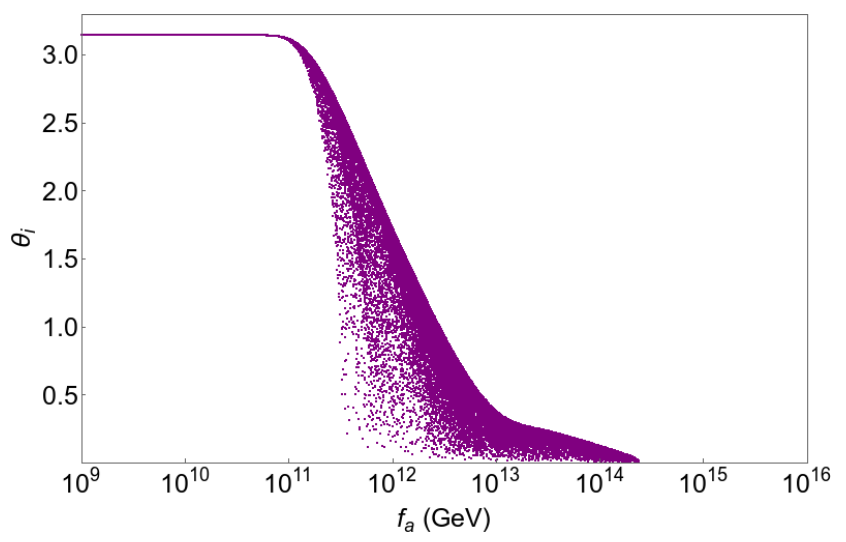

(b)

Figure 2: In a), we plot the relic density of DFSZ axions and higgsino-like WIMPs from a natural SUSY benchmark model using a scan over PQMSSM parameters in the SUSY DFSZ axion model. In $b$ ), we plot the required value of axion misalignment angle $\theta_{i}$ vs. $f_{a}$ such that the calculated relic density of mixed higgsino-axion dark matter matches the measured value $\Omega_{a \widetilde{Z}_{1}} h^{2}=0.12$.

Due to late decay of axinos which occurs after the freeze-out, the neutralino abundance increases to $\Omega_{\widetilde{Z}_{1}} h^{2} \simeq 0.019$. Next, we scan over the following PQMSSM parameter ranges:

- $f_{a}: 10^{9}-10^{16} \mathrm{GeV}$,

- $m_{s}: 1-40 \mathrm{TeV}$,

- $m_{\tilde{a}}: 1-40 \mathrm{TeV}$,

while keeping the gravitino mass fixed at $m_{\tilde{G}}=10 \mathrm{TeV}$ and the reheat temperature fixed at $T_{R}=10^{7} \mathrm{GeV}$.

The mixed axion-higgsino abundance results vs. $f_{a}$ are shown $2^{2}$ in Fig. 2a). The blue dots show the abundance of higgsino-like WIMPs while the green dots show the relic abundance of axions. Blue contour lines show the borders of the WIMP abundance in the allowed region. Red-colored points are excluded by BBN constraints [43] which here occur at very large $f_{a}$ values where saxions are produced at large rates via COs, but then decay late due to couplings suppressed by large $f_{a}$. The brown points are also excluded due to $s \rightarrow a$ a decay which feeds relativistic degrees of freedom into the cosmic plasma so that $\Delta N_{\text {eff }}>1$ [44]. For very low values of $f_{a}$, the higgsino-like WIMPs are at their thermal abundance value since axinos and saxions decay well before neutralino freeze-out. The axions make up the bulk of DM in this case, at the $95 \%$ level. For very low $f_{a}$, this seems artificial since $\theta_{i}$ can be adjusted to very nearly $\pi$ and the axion field would have to sit close to the maximum of its potential. This can

\footnotetext{
${ }^{2}$ In accord with the Particle Data Book [42], we take $f_{a} \equiv f_{A} \times N_{\text {DW }}$ where the domain-wall number $N_{\text {DW }}=6$ for the DFSZ model.
} 
be seen from frame $b$ ) where we show the value of $\theta_{i}$ which is required to enforce $\Omega_{a \widetilde{Z}_{1}} h^{2}=0.12$ vs. $f_{a}$.

As $f_{a}$ increases to nearly $10^{11} \mathrm{GeV}$, then some axinos start decaying after neutralino freezeout thus generating in addition a non-thermal population of WIMPs. If enough WIMPs are injected via decays, then the WIMPs may re-annihilate at the (lower) axino (or saxion) decay temperature which still leads to an increased non-thermal WIMP abundance [27]. For some parameter space points, the non-thermal WIMP production pushes the WIMP abundance above the meaured value $\Omega_{\widetilde{Z}_{1}} h^{2}>0.12$ and so the points become excluded. Also, for $f_{a} \gtrsim 10^{11} \mathrm{GeV}$, the required axion misalignment angle $\theta_{i}$ begins dropping into a more realistic range.

For yet higher values of $f_{a} \gtrsim 10^{13} \mathrm{GeV}$, the minimal WIMP relic density begins decreasing (somewhat buried beneath the green axion points). This is due to increasing CO-production of saxions followed by their late decays. In the case shown here, the saxion couples to axions and axinos via

$$
\mathcal{L} \ni \frac{\xi_{s}}{f_{a}} s\left[\left(\partial_{\mu} a\right)^{2}+i \overline{\tilde{a}} \not \partial \tilde{a}\right]
$$

where the factor $\xi_{s}$ denotes the model dependence of the saa and $s \tilde{a} \tilde{a}$ couplings [24]. The value $\xi_{s}$ can vary between $0-1$ with perhaps some theory prejudice for $\xi_{s} \sim 1$ (which we adopt here). For $\xi_{s} \sim 1$, then the saxion dominantly decays to $a a$ or if kinematically allowed, at comparable rates into $\tilde{a} \tilde{a}$. The $s$ may also decay into SUSY particles and SM particles- complete decay rates and example branching fractions are shown in Ref. [45]. Now back to the dip in Fig. $2 a$ ). These dip points occur for parameter values with $m_{s}<2 m_{\tilde{a}}$ where saxion decays as $s \rightarrow a a$ dominantly but also with substantial $s \rightarrow$ SM particles. The latter decays inject entropy into the cosmic plasma such as to dilute all relics present. Thus, the dip occurs because of saxion decay induced entropy dilution. For yet higher values of $f_{a}$, the minimal value of $\Omega_{\widetilde{Z}_{1}} h^{2}$ turns up again as the delayed saxion decay into WIMPs wins out over entropy dilution. For $f_{a} \gtrsim 10^{14}$ $\mathrm{GeV}$, then always too much WIMP DM is produced and the value of $f_{a}$ is disallowed. Actually, this very high $f_{a}$ range becomes triply disallowed because also saxions begin decaying after the onset of BBN, thus violating limits on late-decaying neutral cosmic particles [43] (red points) and also too many relativistic axions are injected into the cosmic plasma thus violating CMB bounds on extra species of relativistic particles (parametrized in terms of limits on the effective additional neutrino species [42, 44] which we take conservatively as $\Delta N_{\text {eff }}>1$ (brown points)).

For $f_{a} \gtrsim 10^{12} \mathrm{GeV}$, then always the WIMP abundance is non-thermally elevated and many more points are excluded by generating too much WIMP dark matter. A new set of constraints now also impact the PQMSSM parameter space: as the WIMP abundance increases, then the fraction of WIMP dark matter $\xi \equiv \Omega_{\widetilde{Z}_{1}} h^{2} / 0.12$ increases and even though $\xi<1$, the WIMPs may come into conflict with spin-independent (SI), spin-dependent (SD) and indirect WIMP detection (IDD) constraints [32. It is worth noting that we assume the WIMP fraction $\xi$ is the same in the whole universe. In Fig. 3, we plot in $a)$ the value of $\xi \sigma^{\mathrm{SI}}\left(\widetilde{Z}_{1}, p\right)$ and $\left.b\right)$ $\xi \sigma^{\mathrm{SD}}\left(\widetilde{Z}_{1}, p\right)$ versus the value of $\xi$ and in $\left.c\right)$ we plot $\xi^{2}\langle\sigma v\rangle$ versus $\xi$. From frame $\left.a\right)$, we see that as $\xi$ increases, the value of $\xi \sigma^{\mathrm{SI}}\left(\widetilde{Z}_{1}, p\right)$ approaches and then exceeds the latest constraint from the LUX experiment [46]. The locus of the thermal value of $\xi$ for our benchmark point is denoted by a red star. Once $\xi>0.53$, then the parameter space point violates the LUX limit on SI direct WIMP detection. From frame $b$ ), we see that as $\xi$ increases, then the value of $\xi \sigma^{\mathrm{SD}}\left(\widetilde{Z}_{1}, p\right)$ always stays below the most constraining SD limit which is currently from the 

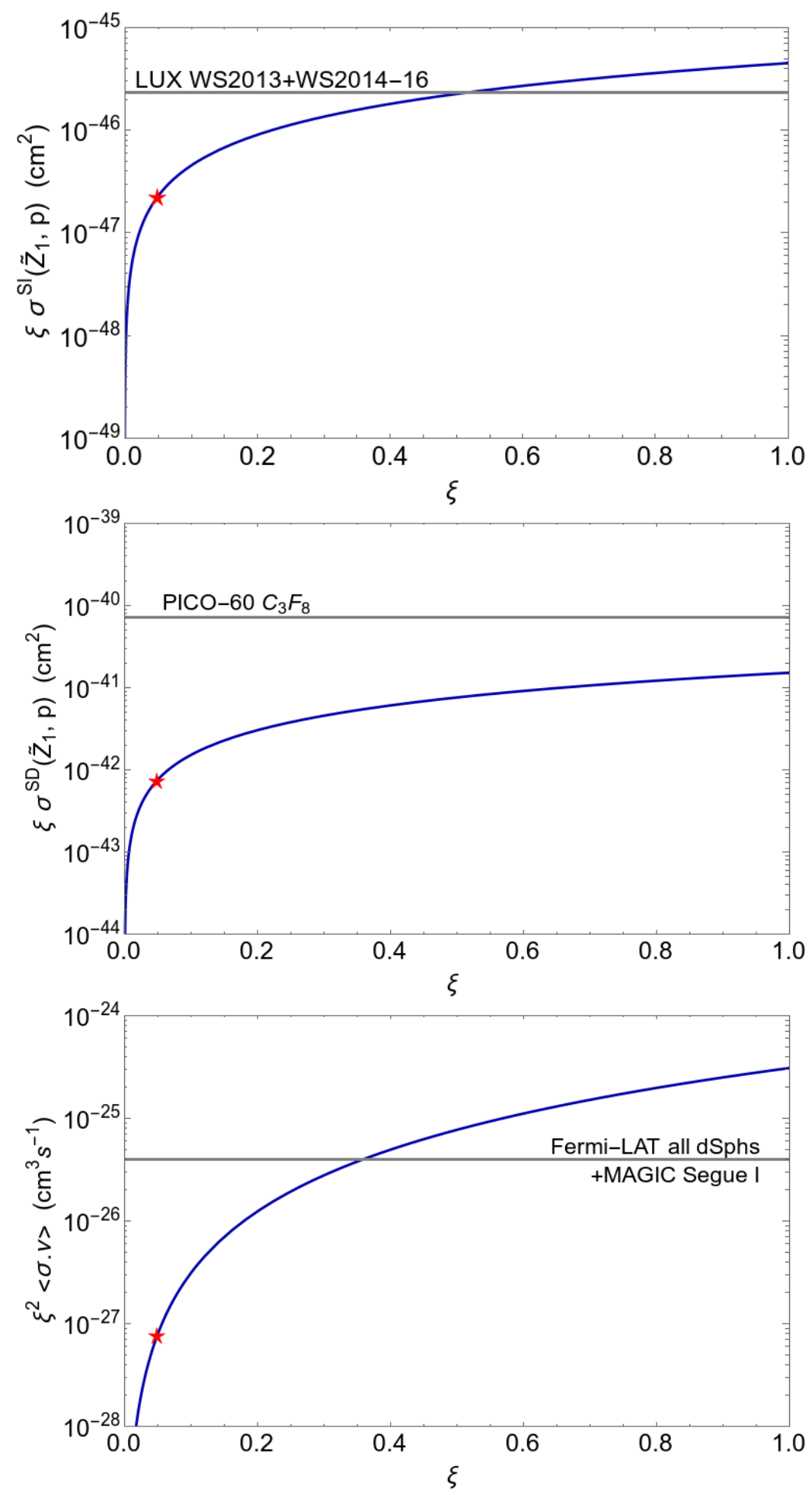

Figure 3: In $a$ ), we plot $\xi \sigma^{\mathrm{SI}}\left(\widetilde{Z}_{1}, p\right)$ vs. $\xi \equiv \Omega_{\widetilde{Z}_{1}} h^{2} / 0.12$ for our natural SUSY benchmark point. The red star denotes the value of $\xi$ obtained from thermal WIMP production only. The horizontal line denotes the upper limit reported from the LUX experiment. In $b$ ), we plot $\xi \sigma^{\mathrm{SD}}\left(\widetilde{Z}_{1}, p\right)$ along with the upper bound from PICO and in $\left.c\right)$ we plot $\xi^{2}\langle\sigma v\rangle$ with an upper limit from Fermi-LAT/MAGIC searches for $\gamma$ emissions from dwarf spheroidal galaxies. 
PICO hot liquid bubble experiment [47]. In frame $c$ ), we find that as $\xi$ increases, now the value of $\xi^{2}\langle\sigma v\rangle$ exceeds limits from Fermi-LAT/MAGIC searches [4] for gamma-ray emissions from WIMP-WIMP annihilation to $W^{+} W^{-}$(this annihilation channel is most relevant for higgsinolike WIMPs) for a value of $\xi \gtrsim 0.35$. Thus, frame $c$ ) in IDD offers the most constraining limit on $\xi$.

An overview of the ultimate allowed region of $f_{a}$ in the natural SUSY DFSZ model is shown in Fig. 4a) where we present a blown-up version of Fig. 2a). In this case, the purpleshaded region denotes values of $\Omega_{\widetilde{Z}_{1}} h^{2}$ which are in violation of the Fermi-LAT/MAGIC dwarphspheroidal constraint. The black points (scanning over $1 \mathrm{TeV}<m_{s}<30 \mathrm{TeV}$ ) with $\Omega_{\widetilde{Z}_{1}} h^{2} \lesssim$ 0.45 are thus fully allowed. The yellow points are seemingly allowed as well, but these points require $\theta_{i}>3$ and seem rather implausible. The allowed region of $f_{a}$ thus breaks up into two disjoint bands: the first runs from $10^{11} \mathrm{GeV}<f_{a}<4 \times 10^{12} \mathrm{GeV}$. Then a gap for values $4 \times 10^{12} \mathrm{GeV}<f_{a}<3 \times 10^{13} \mathrm{GeV}$ ensues where WIMP production is sufficiently large that Fermi-LAT/MAGIC constraints dis-allow the parameter space. For $3 \times 10^{13} \mathrm{GeV}<f_{a}<10^{14}$ $\mathrm{GeV}$, then the $f_{a}$ values are re-allowed due to the effect of saxion entropy dilution of the WIMP abundance (and where WIMP production via $s \rightarrow \tilde{a} \tilde{a}$ is kinematically forbidden). The orange colored points denote where $m_{s} \gtrsim 30 \mathrm{TeV}$ showing that most of this region comes from very heavy saxions. For heavy enough saxions, then the saxion decay rate is enhanced which helps avoid WIMP overproduction by late decays. For even higher $f_{a}>10^{14} \mathrm{GeV}$ values, then all of parameter space is disallowed.

In Fig. $4 b$ ), we present the axion density so that $\Omega_{a} h^{2}+\Omega_{\widetilde{Z}_{1}} h^{2}=0.12$. Red dots show the axion density which satisfy the relic density constraint along with WIMPs but excluded from the indirect searches for overestimating the WIMP abundance (correspond to the purple-shaded region in Fig. 4a)). Allowed parameter space is shown by the green dots where axions make up more than $75 \%$ of the total dark matter density.

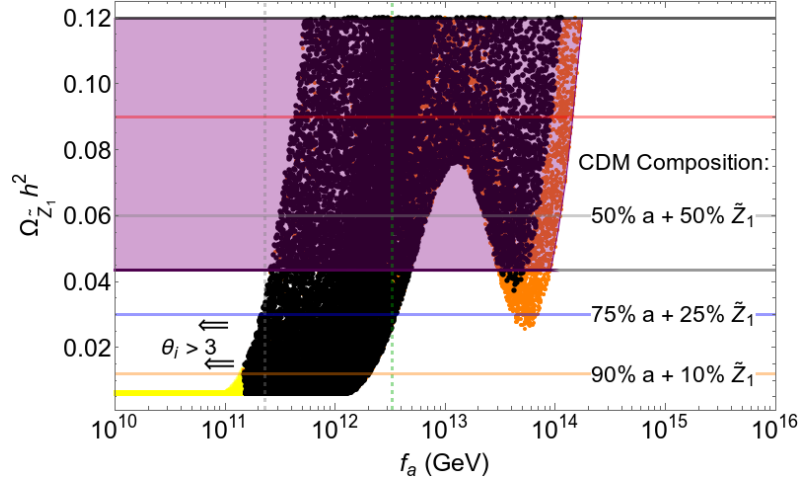

(a)

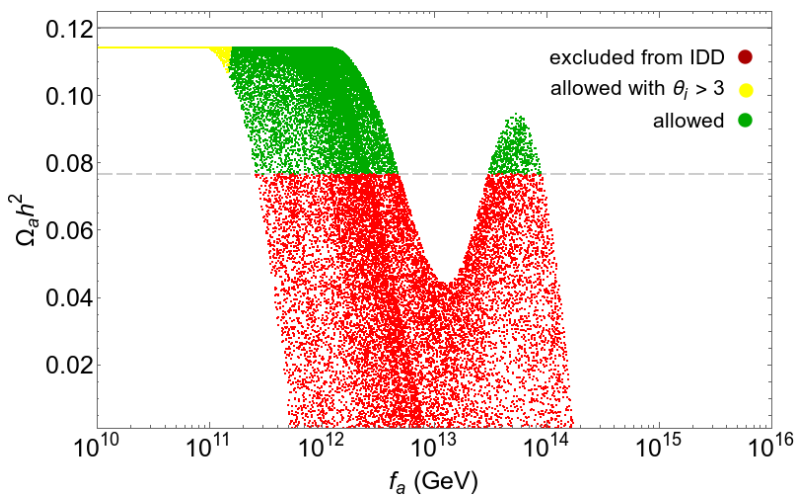

(b)

Figure 4: Relic density of (a) higgsino-like WIMPs and (b) axions from a natural SUSY benchmark model with a scan over PQMSSM parameters in the SUSY DFSZ axion model. In (a), the purple shaded region is excluded by Fermi-LAT/MAGIC limits on WIMP annihilation to gamma ray production in dwarph spheroidal galaxies. Black dots denote where $1 \mathrm{TeV}<m_{s}<30 \mathrm{TeV}$ while orange dots denote where $30 \mathrm{TeV}<m_{s}<40 \mathrm{TeV}$ which enhances entropy dilution from saxion decay. Yellow dots denote where $\theta_{i}>3$. 


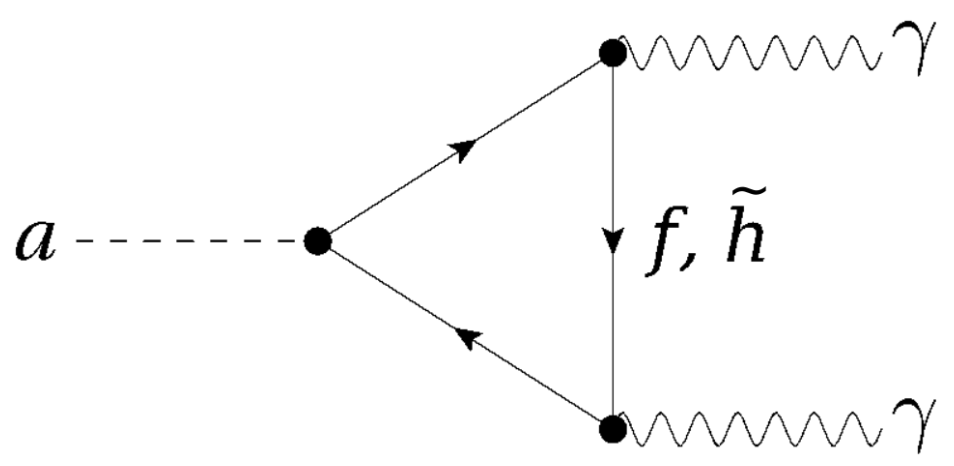

Figure 5: Feynman diagram showing the fermionic loop contributions to the $a-\gamma-\gamma$ coupling.

\section{Axion detection at microwave cavity experiments}

Next, we address the prospects for axion detection in the natural SUSY DFSZ model. At present, the most sensitive experiment searching for QCD axions is the Axion Dark Matter search eXperiment, or ADMX [33, 34]. ADMX implements a super-cooled microwave cavity (Sikivie) detector [49] which can be tuned over a range of frequencies to search for axion-tophoton conversion in the presence of a strong $\vec{B}$-field. The power produced in the cavity at a frequency $\nu_{a}$ corresponding to an axion mass $m_{a}$ is given by

$$
P=g_{a \gamma \gamma}^{2} \frac{\rho_{a}}{m_{a}} B_{0}^{2} V C_{m n p} Q_{L}
$$

where $g_{a \gamma \gamma}$ is the (model dependent) axion-photon-photon coupling, $\rho_{a}$ is the axion local density, $B_{0}$ is the magnetic field strength, $V$ is the volume of the cavity and $Q_{L}$ is the loaded quality factor of the cavity. $C_{m n p}$ denotes a normalized coupling form factor of the axion to a specific frequency mode. The relevant quantities of theoretical interest are then the axion mass $m_{a}$, the axion local density $\rho_{a}$ and the axion coupling $g_{a \gamma \gamma}$. Whereas usually the axion local density is taken to be the same as the DM local density, in our case since axions only make up a portion of the dark matter: then we define $\xi_{a} \equiv \Omega_{a} h^{2} / 0.12$ which gives the fractional axion dark matter density around the earth. Also, we remind the reader that [50]

$$
m_{a}=\frac{z^{1 / 2}}{1+z} \frac{f_{\pi} m_{\pi}}{\left(f_{a} / N_{\mathrm{DW}}\right)} \simeq 6 \mu \mathrm{eV} \frac{10^{12} \mathrm{GeV}}{\left(f_{a} / N_{\mathrm{DW}}\right)}
$$

where $z=m_{u} / m_{d} \simeq 0.56$ but with a plausible range $z: 0.38-0.58$ [42].

The axion coupling strength is given by [9, 42, 51, 52]

$$
g_{a \gamma \gamma}=\frac{\alpha}{2 \pi\left(f_{a} / N_{\mathrm{DW}}\right)}\left(\frac{E}{N}-\frac{2}{3} \frac{4+z}{1+z}\right)
$$

where $E$ and $N$ are the electromagnetic and color anomalies of the axion axial current.

The second contribution in parenthesis of Eq. 12 (from chiral symmetry) gives a value $\sim-1.96$ for $z=0.56$. The anomaly contribution $E / N$ arises from fermions circulating in Fig. 5 ; 
$E=\sum_{\text {fermions }} Q_{\mathrm{em}}^{2} \times n_{\text {col }} \times n_{\text {gen }} \times Q_{\mathrm{PQ}}$. For the non-SUSY KSVZ axion model with uncharged $\left(Q_{\mathrm{em}}=0\right)$ heavy color triplets circulating, then $E / N=0$ so that $g_{a \gamma \gamma}^{\mathrm{KSVZ}} \simeq-1.96 \alpha / 2 \pi\left(f_{a} / N_{\mathrm{DW}}\right)$. For the non-SUSY DFSZ model, then $Q_{\mathrm{PQ}}(Q, L)=+1, Q_{\mathrm{PQ}}\left(U^{c}, D^{c}, E^{c}\right)=0$ so for $(u, c, t)$ quarks we obtain $E=4$ and for $(d, s, b)$ quarks we obtain $E=1$ and for $e, \mu, \tau$ leptons we obtain $E=3$. The sum yields $E / N=8 / 3$ or $g_{a \gamma \gamma}^{\mathrm{DFSZ}} \simeq 0.7 \alpha / 2 \pi\left(f_{a} / N_{\mathrm{DW}}\right)$. For our case of the SUSY DFSZ axion model, we must also add in the higgsino contribution to $g_{a \gamma \gamma}$ with

$Q_{\mathrm{PQ}}\left(\widetilde{H}_{u}, \widetilde{H}_{d}\right)=-1$ so that $E_{\tilde{h}}=-2$. Summing over fermions yields $E / N=6 / 3$ so that there is a near cancellation between the anomaly and chiral symmetry contributions: $g_{a \gamma \gamma}^{\text {SUSY DFSZ }} \simeq$ $0.04 \alpha / 2 \pi\left(f_{a} / N_{\mathrm{DW}}\right)$ for $z=0.56$. In fact, if $z$ is identically 0.5 , then an exact cancellation occurs and the coupling drops to zero (such a diminished $a \gamma \gamma$ coupling has been noted previously for non-SUSY models in Ref's [51]). In such a case, apparently the invisible axion becomes again invisible with respect to its coupling to photons.

The non-SUSY KSVZ, non-SUSY DFSZ and SUSY DFSZ coupling are shown in Fig. 6 in the $m_{a}$ vs. $\sqrt{\xi_{a}} \cdot\left|g_{a \gamma \gamma}\right|$ plane. We also show the ADMX published limits [34] in dark blue and ADMX proposed future search region as light blue. The latter extends down to the non-SUSY DFSZ coupling strength. We also show the value of $\xi_{a} \cdot\left|g_{a \gamma \gamma}\right|$ obtained from our natural SUSY benchmark point with a scan over SUSY DFSZ parameters. The green dots show allowed points whilst the red points are excluded by IDD WIMP search results from FermiLAT. These latter points have a diminished axion abundance since the WIMP abundance is non-thermally-enhanced. The green allowed points actually break into two disjoint regions: the lower $m_{a}$ region corresponds to large $f_{a}$ where CO-produced saxions bring the higgsino-like WIMP abundance into accord with the measured DM relic density via entropy dilution. The larger $m_{a}$-allowed region corresponds to the $f_{a} \sim 10^{11} \mathrm{GeV}-3 \times 10^{12} \mathrm{GeV}$ region of Fig's 2 and 4. The current ADMX search region lies in the intermediate disallowed region where thermal axino production and decay contributes to non-thermal higgsino-like WIMP production which lies in the IDD-constrained region. The SUSY DFSZ coupling strength lies a factor 17 below the non-SUSY DFSZ projection. The non-SUSY DFSZ line has recently been reached in actual ADMX searches [53]. The yellow points at very large $m_{a}>3 \times 10^{-4} \mathrm{eV}$ seem implausible due to some tuning required for $\theta_{i} \simeq \pi$.

A new detection method has been proposed in Ref. [54] that is sensitive to the QCD axion mass $\left(m_{a} \sim\left[10^{-14}, 10^{-6}\right] \mathrm{eV}\right)$ predicted by string/GUT inspired axion dark matter models. Although the proposed experiment is projected to probe $g_{a \gamma \gamma}$ down to $10^{-19} \mathrm{GeV}^{-1}$, the targeted parameter space does not probe our allowed region.

\section{Conclusions}

In this paper we have reported on prospects for axion detection in a model which allows for

- naturalness in the EW sector via SUSY and

- naturalness in the QCD sector via inclusion of PQ symmetry and its concommitant axion.

We specialize to the SUSY DFSZ axion model since this allows for both

- a solution to the SUSY $\mu$ problem (why the superpotential $\mu$ parameter is weak scale rather than Plank scale) and 


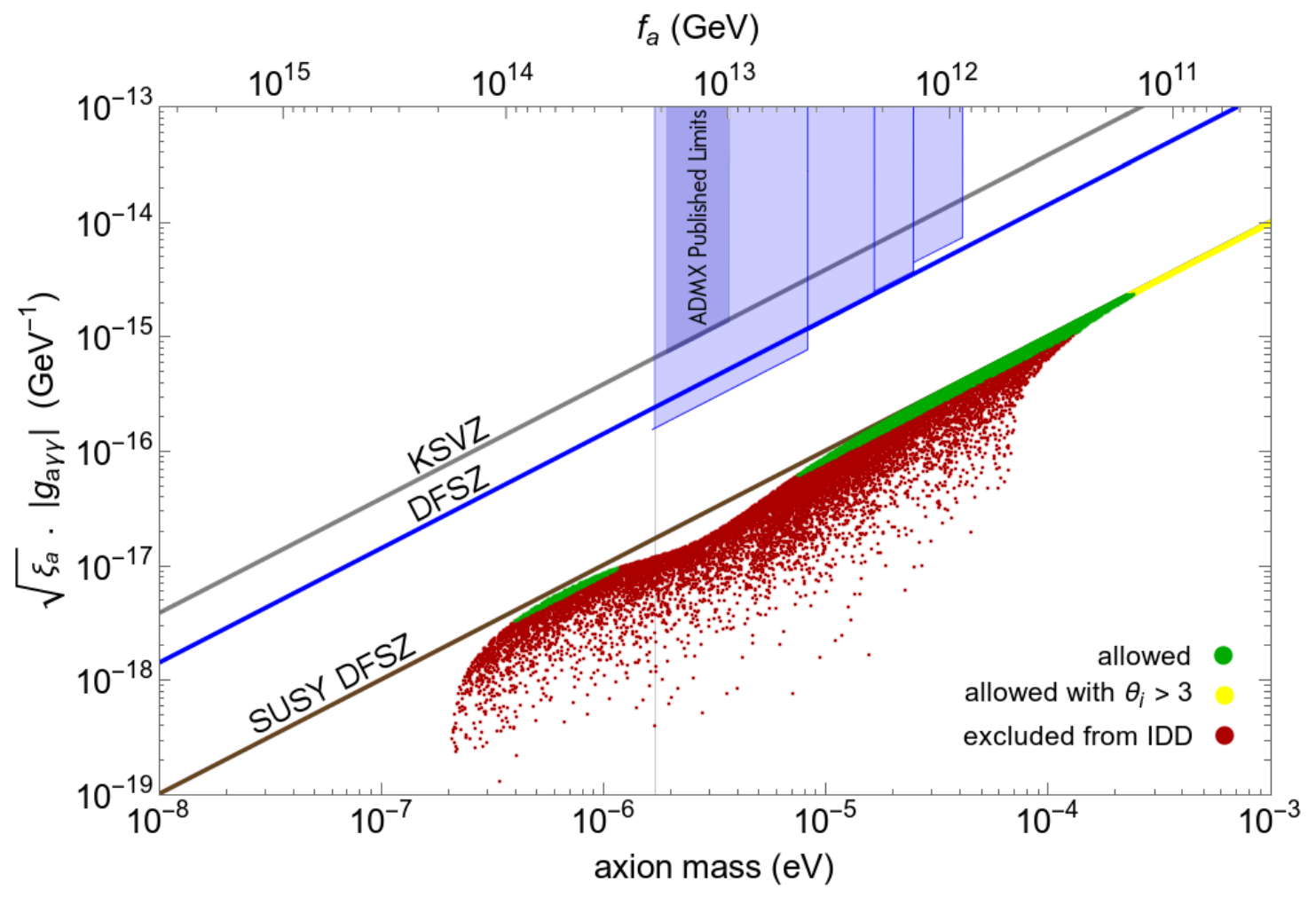

Figure 6: Axion detection rates at microwave cavity experiments in terms of the axion coupling $\left|g_{a \gamma \gamma}\right|$ vs. $m_{a}$. The vertical axis includes a factor $\sqrt{\xi_{a}}$ where $\xi_{a} \equiv \Omega_{a} h^{2} / 0.12$ to account for the depleted abundance of axions. The green points are allowed from natural SUSY while red points are excluded by Fermi-LAT constraints on higgsino-like WIMP annihilation into gamma rays. We also plot lines of SUSY and non-SUSY coupling strengths and current and projected ADMX search regions. The yellow dots are regarded as unnatural since they would require an axion misalignment angle $\theta_{i}>3$. 
- allows for the required Little Hierarchy $\mu \sim 100-300 \mathrm{GeV} \ll m_{\mathrm{SUSY}} \sim 1-10 \mathrm{TeV}$.

A subset of these models actually allows for radiative PQ breaking where the PQ scale $f_{a}$ is generated from SUSY breaking. Such models tend to generate a value $\mu \sim 100-300 \mathrm{GeV}$ from soft SUSY breaking terms of order $1-10 \mathrm{TeV}$ [30]. For these reasons, we feel the model explored in this paper is the most highly motivated axion model available so that the results presented here should be regarded more as a paradigm of what is to be expected for axion physics, rather than some implausible outlier model.

Our main result is summarized in Fig. 6. This plot shows a wide range of $m_{a}$ values over which an axion might be expected. The two allowed regions are disjoint: the upper region with $m_{a}: 10^{-5}-3 \times 10^{-4} \mathrm{eV}$ occurs with mainly axion cold dark matter and a smaller contribution of higgsino-like WIMPs. It is somewhat higher in $m_{a}$ values than is currently being explored at ADMX. The lower region with $m_{a}: 3 \times 10^{-7}-1.5 \times 10^{-6} \mathrm{eV}$ occurs due to CO production of (very heavy) saxions followed by decays to SM (and other) particles which leads to entropy dilution of all relics. An intermediate region is actually excluded by Fermi-LAT bounds on WIMP-WIMP annihilation into gamma rays where the WIMP abundance is non-thermally enhanced mainly due to axino/saxion production and decay in the early universe.

What is more distressing is that in the PQ augmented MSSM, then the axion coupling is severely depressed relative to non-SUSY KSVZ or DFSZ via the inclusion of higgsinos in the $g_{a \gamma \gamma}$ triangle coupling. For $z=m_{u} / m_{d}=0.56$, then the coupling is suppressed by a factor around 17 although the coupling could be even more suppressed for $z=0.5$. The detection rate is somewhat reduced as well due to the fact that axions only make up a portion of the dark matter abundance. This necessitates inclusion of a factor $\sqrt{\xi_{a}}$ in the axion coupling vs. $m_{a}$ plot.

While our results were presented for just one SUSY benchmark, it should be noted that they are still rather general since naturalness requires $\mu \sim 100-300 \mathrm{GeV}$ (the closer to $m_{Z}$ the better) while LHC sparticle mass limits and Higgs mass measurement require sparticle masses, especially gluinos and squarks, in the multi-TeV range. Generally, all natural models based on the MSSM should look pretty close to our benchmark as far as dark matter physics is concerned.

Ultimately, these results seem to show the axion may exist across a broader mass range than otherwise might be expected. Even so in a SUSY DFSZ scenario, axions from stringy models [55, 56] with $f_{a} \sim 10^{16-18} \mathrm{GeV}$ seem highly unlikely in this regard ${ }^{3}$ since saxion production via COs and very late decay makes model points triply excluded via: 1. over non-thermal-production of WIMPs, 2. injection of relativistic particles $(s \rightarrow a a)$ into the cosmic plasma (violating bounds on $\Delta N_{\text {eff }}$ ) and 3. violations of BBN constraints from late-time saxion decays. Also, our results show the axion signal strength may be far lower than might be expected from non-SUSY axion models due to inclusion of higgsinos circulating in the $a \gamma \gamma$ triangle loop. Thus, axions may be rendered once again more invisible to experiment than otherwise anticipated. As a result, we urge new ideas and initiatives to probe more broadly and more deeply into the $g_{a \gamma \gamma}$ vs. $m_{a}$ axion parameter space.

\footnotetext{
${ }^{3}$ An exception occurs when saxion decays to neutralinos are not kinematically allowed and the $\mu$ term is large so saxions decaying into gauge bosons and into the Higgses are the dominant decay modes [45, 35].
} 


\section{Acknowledgments}

We thank Jihn E. Kim and Seokhoon Yun for discussion and Vernon Barger for a close reading of this manuscript. This work was supported in part by the US Department of Energy, Office of High Energy Physics. The work of KJB was supported by IBS under the project code, IBSR018-D1. The computing for this project was performed at the OU Supercomputing Center for Education \& Research (OSCER) at the University of Oklahoma (OU).

\section{References}

[1] G. F. Giudice, In *Kane, Gordon (ed.), Pierce, Aaron (ed.): Perspectives on LHC physics* 155-178 [arXiv:0801.2562 [hep-ph]]; M. Dine, Ann. Rev. Nucl. Part. Sci. 65 (2015) 43;

[2] R. D. Peccei, Lect. Notes Phys. 741 (2008) 3 [hep-ph/0607268].

[3] G. 't Hooft, Phys. Rev. Lett. 37 (1976) 8; G. 't Hooft, Phys. Rev. D 14 (1976) 3432 Erratum: [Phys. Rev. D 18 (1978) 2199].

[4] S. Weinberg, Phys. Rev. D 11 (1975) 3583.

[5] R. Peccei and H. Quinn, Phys. Rev. Lett. 38 (1977) 1440 and Phys. Rev. D 16 (1977) 1791.

[6] S. Weinberg, Phys. Rev. Lett. 40 (1978) 223; F. Wilczek, Phys. Rev. Lett. 40 (1978) 279.

[7] J. E. Kim, Phys. Rev. Lett. 43 (1979) 103; M. A. Shifman, A. Vainstein and V. I. Zakharov, Nucl. Phys. B 166 (1980) 493.

[8] M. Dine, W. Fischler and M. Srednicki, Phys. Lett. B 104 (1981) 199; A. R. Zhitnitsky, Sov. J. Nucl. Phys. 31 (1980) 260 [Yad. Fiz. 31 (1980) 497].

[9] J. E. Kim, Phys. Rept. 150 (1987) 1; J. E. Kim and G. Carosi, Rev. Mod. Phys. 82 (2010) 557.

[10] G. Aad et al. [ATLAS Collaboration], Phys. Lett. B 716 (2012) 1.

[11] S. Chatrchyan et al. [CMS Collaboration], Phys. Lett. B 716 (2012) 30.

[12] H. Baer and X. Tata, Weak Scale Supersymmetry: From Superfields to Scattering Events, (Cambridge University Press, 2006).

[13] G. Aad et al. [ATLAS Collaboration], Phys. Rev. D 87 (2013) 012008.

[14] S. Chatrchyan et al. [CMS Collaboration], J. High Energy Phys. 1210 (2012) 018.

[15] R. Barbieri and A. Strumia, hep-ph/0007265.

[16] H. Baer, V. Barger, P. Huang, D. Mickelson, A. Mustafayev and X. Tata, Phys. Rev. D 87 (2013) no.11, 115028.

[17] K. L. Chan, U. Chattopadhyay and P. Nath, Phys. Rev. D 58 (1998) 096004 hep-ph/9710473; H. Baer, V. Barger and P. Huang, JHEP 1111 (2011) 031. 
[18] H. Baer, V. Barger, P. Huang, A. Mustafayev and X. Tata, Phys. Rev. Lett. 109 (2012) 161802.

[19] H. Baer, V. Barger and A. Mustafayev, Phys. Rev. D 85 (2012) 075010.

[20] H. Baer, V. Barger and M. Savoy, Phys. Rev. D 93 (2016) no.3, 035016.

[21] H. Baer, V. Barger and D. Mickelson, Phys. Lett. B 726 (2013) 330.

[22] T. Goto and M. Yamaguchi, Phys. Lett. B 276, 103 (1992).

[23] E. J. Chun, J. E. Kim and H. P. Nilles, Phys. Lett. B 287, 123 (1992).

[24] E. J. Chun and A. Lukas, Phys. Lett. B 357 (1995) 43.

[25] K. J. Bae, H. Baer, E. J. Chun and C. S. Shin, Phys. Rev. D 91, no. 7, 075011 (2015).

[26] For a review, see H. Baer, K. Y. Choi, J. E. Kim and L. Roszkowski, Phys. Rept. 555 (2015) 1.

[27] K. Y. Choi, J. E. Kim, H. M. Lee and O. Seto, Phys. Rev. D 77 (2008) 123501; H. Baer, A. Lessa, S. Rajagopalan and W. Sreethawong, JCAP 1106 (2011) 031.

[28] E. J. Chun, Phys. Rev. D 84 (2011) 043509; K. J. Bae, E. J. Chun and S. H. Im, JCAP 1203 (2012) 013.

[29] J. E. Kim and H. P. Nilles, Phys. Lett. B 138 (1984) 150.

[30] H. Murayama, H. Suzuki and T. Yanagida, Phys. Lett. B 291 (1992) 418; K. Choi, E. J. Chun and J. E. Kim, Phys. Lett. B 403 (1997) 209; K. J. Bae, H. Baer and H. Serce, Phys. Rev. D 91 (2015) no.1, 015003

[31] K. J. Bae, H. Baer, V. Barger, M. R. Savoy and H. Serce, Symmetry 7, no. 2, 788 (2015).

[32] H. Baer, V. Barger and H. Serce, Phys. Rev. D 94 (2016) no.11, 115019.

[33] S. J. Asztalos et al. [ADMX Collaboration], Phys. Rev. Lett. 104 (2010) 041301;

[34] G. Rybka [ADMX Collaboration], Phys. Dark Univ. 4 (2014) 14.

[35] K. J. Bae, H. Baer, A. Lessa and H. Serce, JCAP 1410 (2014) no.10, 082.

[36] D. Matalliotakis and H. P. Nilles, Nucl. Phys. B 435 (1995) 115; P. Nath and R. L. Arnowitt, Phys. Rev. D 56 (1997) 2820; J. R. Ellis, T. Falk, K. A. Olive and Y. Santoso, Nucl. Phys. B 652 (2003) 259; H. Baer, A. Mustafayev, S. Profumo, A. Belyaev and X. Tata, JHEP 0507 (2005) 065 .

[37] H. Baer, V. Barger, J. S. Gainer, P. Huang, M. Savoy, H. Serce and X. Tata, arXiv:1702.06588 [hep-ph].

[38] H. Baer, A. Lessa and W. Sreethawong, JCAP 1201 (2012) 036; K. J. Bae, H. Baer and A. Lessa, JCAP 1304 (2013) 041. 
[39] L. F. Abbott and P. Sikivie, Phys. Lett. B 120 (1983) 133; J. Preskill, M. Wise and F. Wilczek, Phys. Lett. B 120 (1983) 127; M. Dine and W. Fischler, Phys. Lett. B 120 (1983) 137; M. Turner, Phys. Rev. D 33 (1986) 889; K. J. Bae, J. H. Huh and J. E. Kim, JCAP 0809 (2008) 005 .

[40] L. Visinelli and P. Gondolo, Phys. Rev. D 80 (2009) 035024.

[41] K. J. Bae, H. Baer, H. Serce and Y. F. Zhang, JCAP 1601 (2016) 012; K. J. Bae, H. Baer, K. Hamaguchi and K. Nakayama, JHEP 1702 (2017) 017.

[42] See A. Ringwald, L. Rosenberg and G. Rybbka in C. Patrignani et al. [Particle Data Group], Chin. Phys. C 40 (2016) no.10, 100001.

[43] K. Jedamzik, Phys. Rev. D 74 (2006) 103509.

[44] P. A. R. Ade et al. [Planck Collaboration], Astron. Astrophys. 594 (2016) A13.

[45] K. J. Bae, H. Baer and E. J. Chun, Phys. Rev. D 89 (2014) no.3, 031701; K. J. Bae, H. Baer and E. J. Chun, JCAP 1312 (2013) 028.

[46] D. S. Akerib et al. [LUX Collaboration], Phys. Rev. Lett. 118 (2017) no.2, 021303.

[47] C. Amole et al. [PICO Collaboration], arXiv:1702.07666 [astro-ph.CO].

[48] M. L. Ahnen et al. [MAGIC and Fermi-LAT Collaborations], JCAP 1602 (2016) no.02, 039.

[49] P. Sikivie, Phys. Rev. Lett. 51 (1983) 1415 Erratum: [Phys. Rev. Lett. 52 (1984) 695].

[50] W. A. Bardeen and S.-H. H. Tye, Phys. Lett. 74B (1978) 229; W. A. Bardeen, R. D. Peccei and T. Yanagida, Nucl. Phys. B 279 (1987) 401.

[51] D. B. Kaplan, Nucl. Phys. B 260 (1985) 215.

[52] M. Srednicki, Nucl. Phys. B 260 (1985) 689.

[53] Talk by D. Tanner at US DoE Cosmic Visions workshop, March, 2017.

[54] Y. Kahn, B. R. Safdi and J. Thaler, Phys. Rev. Lett. 117, no. 14, 141801 (2016) doi:10.1103/PhysRevLett.117.141801 [arXiv:1602.01086 [hep-ph]].

[55] P. Svrcek and E. Witten, JHEP 0606 (2006) 051.

[56] H. Baer and A. Lessa, JHEP 1106 (2011) 027. 